\title{
Semi-lattice of varieties of quasigroups with linearity
}

\author{
F. M. Sokhatsky, H. V. Krainichuk, and V. A. Sydoruk
}

\author{
Communicated by A. V. Zhuchok
}

\begin{abstract}
A BstraCt. A $\sigma$-parastrophe of a class of quasigroups $\mathfrak{A}$ is a class ${ }^{\sigma} \mathfrak{A}$ of all $\sigma$-parastrophes of quasigroups from $\mathfrak{A}$. A set of all pairwise parastrophic classes is called a parastrophic orbit or a truss. A parastrophically closed semi-lattice of classes is a bunch.

A linearity bunch is a set of varieties which contains the variety of all left linear quasigroups, the variety of all left alinear quasigroups, all their parastrophes and all their intersections. It contains 14 varieties, which are distributed into six parastrophic orbits. All quasigroups from these varieties are called dilinear. To obtain all varieties from the bunch, concepts of middle linearity and middle alinearity are introduced. A well-known identity or a system of identities which describes a variety from every parastrophic orbit of the bunch is cited. An algorithm for obtaining identities which describe all varieties from the parastrophic orbits is given. Examples of quasigroups distinguishing one variety from the other are presented.
\end{abstract}

\section{Introduction}

The variety of all quasigroups is parastrophically closed, that is, every parastrophe of a quasigroup is a quasigroup. Thus, every concept given for quasigroups is introduced for all their parastrophes. Therefore, for $n$-ary quasigroups, $n$ ! concepts are defined simultaneously - a concept introduced directly and those concepts, which are transformed from their

2020 MSC: Primary 20N05, 20N15, 39B52, 08A05; Secondary 05A15, $05 B 07$.

Key words and phrases: quasigroup, parastrophe, identity, parastrophic symmetry, parastrophic orbit, truss, bunch, left, right, middle linearity, alinearity, central, semi-central, semi-linear, semi-alinear, linear, alinear variety. 
parastrophes. The analysis of these relations and some general theorems are given in [20] (see also [18]). As an example, the notion of neutrality of elements in quasigroups has been summarized there. In this article, we analyze the concept of linearity of quasigroups and give the corresponding semi-lattice of varieties.

Now, we present a system of notions of quasigroups with some linearity property taken from different articles. Let $(Q ;+, 0)$ be an arbitrary group, $J x:=-x, \alpha, \beta$ be its unitary permutations, i.e. $\alpha 0=\beta 0=0$, and $a \in Q$ and let $x \circ y:=\alpha x+a+\beta y$. Following V.D. Belousov [1], a permutation of a group is called a di-automorphism, if it is an automorphism or an anti-automorphism of the group. The permutations $\alpha, \beta, J \alpha \beta^{-1}$ will be called left, right and middle coefficients respectively. If a left (right, middle) coefficient is an automorphism, then the quasigroup $(Q ; \circ)$ is called left (right, middle) linear or semi-linear. If a left (right, middle) coefficient is an anti-automorphism, then the quasigroup $(Q ; \circ)$ is called left (right, middle) alinear or semi-alinear. All of them are called semidilinear quasigroups. If left and right (left and middle, right and middle) coefficients are automorphisms, then the quasigroup is called left-right (leftmiddle, right-middle) linear. If two coefficients are anti-automorphisms, then the third one is an anti-automorphism as well and the quasigroup is called alinear. If a quasigroup is linear or alinear, then it is called dilinear. Traditionally, a left-right linear quasigroup is called linear. Linear and semilinear quasigroups over commutative groups are called semi-central and central respectively. Middle semi-linear, middle semi-alinear and middle central quasigroups are introduced here for the first time.

A.K. Sushkevich $[27,1937]$ was the first who defined medial quasigroups as quasigroups $(Q ; \cdot)$ satisfying the identity $x y \cdot u v=x u \cdot y v$. The wellknown Bruck-Toyoda theorem can be formulated as follows: "Medial quasigroups are exactly linear quasigroups whose coefficients commute". Later, these quasigroups were studied by many authors (see $[4,16,17,26$, 28]).

For the first time, linear (i.e. left-right linear) quasigroups were introduced by V.D. Belousov $[1,2,1966]$ when he studied balanced identities on quasigroups. He proved that some quasigroups satisfying balanced identities are linear, and vice versa if in a linear quasigroup left and right coefficients have finite orders, then the quasigroups satisfies a balanced identity. But he did not find identities which characterize the class of all linear quasigroups. It was done by G.B. Belyavskaya and A.Kh. Tabarov [7, 1992], moreover they have defined and characterized alinear quasigroups. 
Linear quasigroups defined on abelian groups were introduced by T. Kepka, P. Němec $[13,14,1971]$ and they called them $T$-quasigroups. G.B. Belyavskaya $[8,1989]$ proved that $T$-quasigroups are exactly the quasigroups which coincide with their center. Now, $T$-quasigroups are called central quasigroups. G.B.Belyavskaya also found two identities that characterize the variety of all central quasigroups $[8,1989]$. Later G.B. Belyavskaya $[3,1994]$ proved that abelian algebra (in the sense of R. Freese, R. McKenzie [9]) in the variety of all quasigroups are exactly central quasigroups . G.B. Belyavskaya [5, 2014] researched some identities in quasigroups with 0 -ary operation characterizing quasigroups isotopic to abelian groups.

For the first time, left and right linear and alinear were introduced by A. Kh. Tabarov [29, 1992]. Independently, these notions were investigated by F.M. Sokhatsky. His works [22, 23, 1995], [24, 1996] devoted to group isotopes. In particular, for semi-dilinear and for dilinear quasigroups the following results were obtained:

- some characteristics for a quasigroup identity to have semi-dilinear or dilinear property, i.e., every quasigroup which satisfies the identity is semi-dilinear or dilinear;

- an algorithm for finding formulas which describe isotopical closure of a class of groups. In particular, it is proved that (linear, alinear, semi-linear, semi-dilinear) isotopical closure of a variety of groups is a variety of (linear, alinear, semi-linear, semi-dilinear) quasigroups;

- an algorithm for up to isomorphic description of group isotopes and semi-dilinear quasigroups is given;

- connections among sub-quasigroups and congruences of group isotopes and the corresponding groups have been established.

For every variety of semi-dilinear quasigroups, the obtained algorithm gives a system of identities which characterizes the variety. G.B. Belyavskaya and A. Kh. Tabarov [6, 2007], [28, 2009] have found one identity for each of the varieties (below we cite some of them).

The obtained results about semi-lattice of varieties of quasigroups having some property of linearity are summarized by A.Kh. Tabarov $[28,2009]$, he lists 11 varieties there, but the list is not complete. For example, (23)parastrophe of a left linear quasigroup which is not right linear is neither left linear, nor right linear, but it has some property that is transformed from left linearity. G.B. Belyavskaya [5, 2014] studied parastrophic equivalence of identities in primitive quasigroups and parastrophically equivalent balanced and near-balanced identities characterizing quasigroups isotopic 
to groups (to abelian groups). Considering all parastrophes, we introduce middle linearity and middle alinearity concepts.

A semi-lattice of varieties is called a bunch, if it is parastrophically closed. A set of all pairwise parastrophic varieties is called a parastrophic orbit or a truss. We prove that there are six maximal varieties of quasigroups with linearity that are distributed into two parastrophic orbits: three varieties of semi-linear quasigroups and three varieties of semi-alinear quasigroups (left, right and middle). The linearity bunch consists of 14 varieties distributed into six parastrophic orbits: semi-linear (three varieties), semi-alinear (three varieties), linear (three varieties), alinear (one variety), semi-central (three varieties) and central quasigroups (one variety). For every variety, the system of identities describing the variety is established. Also, examples of quasigroups distinguishing one variety from the other are given.

Other results on quasigroups with linearity property one can find in $[4,5,10-12,15-17,25,26,28]$.

\section{Preliminaries}

A quasigroup is an algebra $(Q ; \cdot, \cdot, \cdot, \cdot)$ satisfying the identities

$$
(x \cdot y)^{\ell} \cdot y=x, \quad\left(x^{\ell} \cdot y\right) \cdot y=x, \quad x^{r} \cdot(x \cdot y)=y, \quad x \cdot\left(x^{r} \cdot y\right)=y .
$$

The operation $(\cdot)$ is called main and $(\stackrel{\ell}{\cdot}),(\stackrel{r}{\cdot})$ are its left and right divisions. These operations and their dual ones which are defined by

$$
x^{s} \cdot y:=y \cdot x, \quad x \cdot s \ell=y^{\ell} \cdot x, \quad x \cdot s r y:=y^{r} \cdot x
$$

are called parastrophes of $(\cdot)$ and the defining identities are called primary. The last three relationships establish bijection among identities of

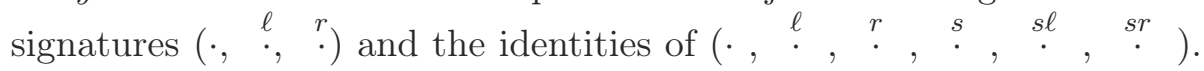
Therefore, throughout of the article we consider identities on quasigroups

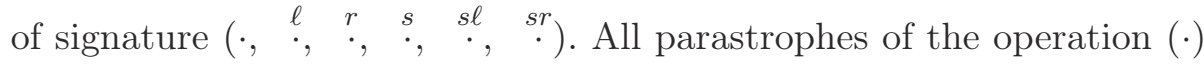
can be defined by:

$$
x_{1 \sigma}{ }^{\sigma} x_{2 \sigma}=x_{3 \sigma}: \Leftrightarrow x_{1} \cdot x_{2}=x_{3},
$$

where $\sigma \in S_{3}:=\{\iota, \ell, r, s, s \ell, s r\}, s:=(12), \ell:=(13), r:=(23)$. It is easy to verify that

$$
{ }^{\sigma}(\tau)=\left({ }^{\sigma \tau} \cdot\right)
$$

holds for all $\sigma, \tau \in S_{3}$. 
A transformation of an identity $\mathfrak{i d}$ to identity $\mathfrak{i d} \mathfrak{d}^{\prime}$ using primary identities is called primary (parastrophic) transformation [19]. A transformation of the identity $\mathfrak{i} \mathfrak{d}$ to the identity ${ }^{\sigma} \mathfrak{i d}$ is called a parastrophic transformation ( $\sigma$-parastrophic transformation), if it can be obtained by replacing the main operation with some its $\sigma^{-1}$-parastrophe [20].

$\sigma$-parastrophe of a class $\mathfrak{A}$ of quasigroups is called a class ${ }^{\sigma} \mathfrak{A}$ consisting of all $\sigma$-parastrophes of quasigroups from $\mathfrak{A}$.

Let $P$ be an arbitrary proposition in a class of quasigroups $\mathfrak{A}$. The proposition ${ }^{\sigma} P$ is said to be a $\sigma$-parastrophe of $P$, if it can be obtained from $P$ by replacing every $\tau$-parastrophe with $\tau \sigma^{-1}$ for every $\tau \in S_{3}$.

Theorem 1. [20,21] Let $\mathfrak{A}$ be a class of quasigroups, then a proposition $P$ is true in $\mathfrak{A}$ if and only if ${ }^{\sigma} P$ is true in ${ }^{\sigma} \mathfrak{A}$.

For instance, let a variety of quasigroups $\mathfrak{B}$ is defined by an identity

$$
\left(x^{r} \cdot y\right)^{s \ell} \cdot(y \cdot x)=y^{\ell} \cdot x .
$$

By this theorem, $s \ell$-parastrophe ${ }^{s \ell} \mathfrak{B}$ of $\mathfrak{B}$ is described by

$$
\left(x^{r(s \ell)^{-1}} y\right)^{s \ell(s \ell)^{-1}}\left(y^{(s \ell)^{-1}} x\right)=y^{\ell(s \ell)^{-1}} x .
$$

Since $r(s \ell)^{-1}=r s r=\ell, \ell(s \ell)^{-1}=\ell s r=s r r=s$, the identity is

$$
\left(x^{\ell} \cdot y\right) \cdot\left(y^{s r} \cdot x\right)=y^{s} \cdot x .
$$

Thus, the variety ${ }^{s \ell} \mathfrak{B}$ is described by $\left(x^{\ell} \cdot y\right) \cdot\left(x^{r} \cdot y\right)=x y$.

Two identities are called:

1) equivalent, if they define the same variety;

2) primarily equivalent, if one of them can be obtained from the other in a finite number of applications of primary identities (1)-(2) (primary equivalent identities are equivalent);

3) $\sigma$-parastrophic, if one of them can be obtained from the other by $\sigma$-parastrophic transformation;

4) $\sigma$-parastrophically equivalent, if they define $\sigma$-parastrophic varieties (according to Theorem 1, $\sigma$-parastrophically equivalent identities define $\sigma$-parastrophic varieties);

5) $\sigma$-parastrophically primarily equivalent, if one of them can be obtained in a finite number of applications of primary identities and $\sigma_{1}$-, $\sigma_{2}-, \ldots, \sigma_{k^{-}}$parastrophic transformations such that $\sigma_{1} \sigma_{2} \ldots \sigma_{k}=\sigma$ for some $k \in \mathbb{N}$.

In a generalized case $\sigma$ will be omitted. For example, two identities are called parastrophically equivalent, if they are $\sigma$-parastrophically equivalent for some $\sigma \in S_{3}$. 


\subsection{Canonical decompositions of group isotopes}

In this subsection, we give more precise definitions of canonical decomposition of group isotopes.

Definition 1. A groupoid $(Q ; \cdot)$ is called an isotope of $\left(Q^{\prime} ;+\right)$, if there exists a triplet of bijections $(\delta, \nu, \gamma)$ which is called an isotopism such that $x \cdot y:=\gamma\left(\delta^{-1} x+\nu^{-1} y\right)$ for all $x, y \in Q$. An isotope of a group is called a group isotope. A permutation $\alpha$ of a set $Q$ is called unitary of a group $(Q ;+, 0)$, if $\alpha(0)=0$.

Definition 2. [23] Let $(Q ; \cdot)$ be a group isotope and 0 be an arbitrary element of $Q$, then the right part of the formula

$$
x \cdot y=\alpha x+a+\beta y
$$

is called a 0 -canonical decomposition, if $(Q ;+)$ is a group, 0 is its neutral element and $\alpha 0=\beta 0=0$.

In this case: the element 0 is a defining element; $(Q ;+)$ is a decomposition group; $a$ its free member; $\alpha$ its left, i.e., 2-coefficient; $\beta$ its right, i.e., 1-coefficient; $J \gamma$, where $\gamma:=\alpha \beta^{-1}$, is its middle, i.e., 3-coefficient. Briefly, the canonical decomposition will be denoted by $(+, 0, \alpha, \beta, a)$.

Theorem 2. [23] An arbitrary element $b$ uniquely defines a b-canonical decomposition of an arbitrary group isotope.

Let $(5)$ be a canonical decomposition of an isotope $(Q ; \cdot)$ of an arbitrary group $(Q ;+)$. Then it is easy to prove (see $[4,22])$ that all its parastrophes have the following forms

$$
\begin{array}{ll}
x^{\iota} \cdot y:=\alpha x+a+\beta y ; & x \stackrel{s}{\bullet} y=\beta x+a+\alpha y ; \\
x^{\ell} \cdot y=\alpha^{-1}(x-\beta y-a) ; & x \cdot s y=\alpha^{-1}(y-\beta x-a) ; \\
x^{r} \cdot y=\beta^{-1}(-a-\alpha x+y) ; & x \cdot y=\beta^{-1}(-a-\alpha y+x) .
\end{array}
$$

Remark 1. Let $(Q ;+, 0)$ be a group, $J x:=-x$ its inverse-valued operation and $\theta$ its automorphism and $\varphi$ be a permutation of $Q$. Then $J_{\varphi}:=\varphi^{-1} J \varphi$ is the inverse-valued operation and $\theta_{\varphi}:=\varphi^{-1} \theta \varphi$ is an automorphism of $\varphi^{-1}$-isomorphe which is denoted by $(Q ; \underset{\varphi}{+})$ and defined by

$$
x_{\varphi}+y:=\varphi^{-1}(\varphi x+\varphi y) .
$$

$\varphi^{-1}$-isomorphe of $(Q ;+, 0)$ has the same neutral element 0 , if $\varphi 0=0$, and $\theta_{\theta}=\theta$. 


\subsection{Actions}

Let $S_{3}:=\{\iota, s, \ell, r, s \ell, s r\}$ denote the symmetric group of the set $\{1,2,3\}$, where $s:=(12), \ell:=(13), r:=(23)$. Let $S_{3}$ act on a set $K$, i.e. for all $k \in K$ and for all $\sigma \in S_{3}$ the element ${ }^{\sigma} k$ belongs to $K$ and equalities ${ }^{\sigma}\left({ }^{\tau} k\right)={ }^{\sigma \tau} k,{ }^{\iota} k=k$ hold. Elements ${ }^{\sigma} k$ and $m$ are called $\sigma$ parastrophic and $\sigma$ is a parastrophism. An orbit of an element $k \in K$ is called a parastrophic orbit or a truss of $k$ and is denoted by $\operatorname{Po}(k)$ (in previous articles a truss of $k$ is denoted by $\operatorname{Tr}(k)$ ); a stabilizer group of $k$ will be called a parastrophic symmetry group of $k$ and is denoted by $\operatorname{Ps}(k)$.

\begin{tabular}{|c|c|c|c|}
\hline $\mathrm{Po}(k)$ & Element $k$ & $\operatorname{Ps}(k)$ & Quantity \\
\hline totally symm. & totally symm., & $\operatorname{Ps}(k)=S_{3}$ & $|\mathrm{Po}(k)|=1$ \\
\hline semi-symmetry & semi-symm., & $\operatorname{Ps}(k) \supseteq A_{3}$ & $|\mathrm{Po}(k)|=1,2$ \\
\hline \multirow{3}{*}{$\begin{array}{l}\text { single } \\
\text { symmetry }\end{array}$} & middle symm., & $\operatorname{Ps}(k) \supseteq\{\iota, s\}$ & $|\mathrm{Po}(k)|=1,3$ \\
\hline & left symm., & $\overline{\operatorname{Ps}}(k) \supseteq\{\iota, r\} ;$ & $|\operatorname{Po}(k)|=1,3 ;$ \\
\hline & right symm., & $\operatorname{Ps}(k) \supseteq\{\iota, \ell\}$ & $|\mathrm{Po}(k)|=1,3$ \\
\hline asymmetry & asymmetric, & $\operatorname{Ps}(k)=\{\iota\}$ & $|\mathrm{Po}(k)|=6$. \\
\hline
\end{tabular}

\section{Linearity of isotopes of groups}

Actually, every quasigroup with linearity is a group isotope. In this section we define these quasigroups using a canonical decomposition of group isotopes and prove that the concept of linearity does not depend on the decomposition.

Definition 3. An isotope of an arbitrary group with a canonical decomposition (5) is called

- $i$-linear, if the $i$-coefficient is an automorphism of the decomposition group, where $i=1,2,3$;

- $i$-alinear, if the $i$-coefficient is an anti-automorphism of the decomposition group, where $i=1,2,3$;

- semi-linear or single linear, if it is $i$-linear for some $i=1,2,3$;

- semi-alinear or single alinear, if it is $i$-alinear for some $i=1,2,3$;

- $i j$-linear, if it is $i$-linear and $j$-linear for some $i \neq j$;

- linear, if it is $i$-linear for all $i \in\{1,2\}$;

- alinear, if it is $i$-alinear for all $i \in\{1,2\}$.

Dilinear property means corresponding linear or alinear property [1, p.56].

Hence, a group isotope is: left linear (alinear), if the left coefficient of its canonical decomposition is an automorphism (anti-automorphism) of the decomposition group; linear, if it is left and right linear. There are 
six classes of semi-dilinear quasigroups: left, right and middle semi-linear quasigroups and left, right and middle alinear quasigroups.

Proposition 1. The definitions of left, right and middle linearity given above are correct.

In other words, they do not depend on canonical decompositions of the corresponding group isotopes.

Proof. Let $(+, 0, \alpha, \beta, a)$ and $\left(\circ, e, \alpha_{1}, \beta_{1}, b\right)$ be canonical decompositions of a group isotope $(Q ; \cdot)$. A dependence between them is the following (see [23]):

$$
\begin{array}{cc}
x \circ y=x-e+y, & \alpha_{1} x=\alpha x-\alpha e+e, \\
\beta_{1} y=e-\beta e+\beta y, & b=\alpha e+a+\beta e .
\end{array}
$$

To prove independence of the left linearity concept on canonical decompositions, we have to prove that $\alpha_{1}$ is an automorphism of the group $(Q ; \circ, e)$ if and only if $\alpha$ is an automorphism of the group $(Q ;+, 0)$. The statement " $\alpha_{1}$ is an automorphism of $(Q ; \circ, e)$ " means that for all $x, y \in Q$

$$
\alpha_{1}(x \circ y)=\alpha_{1} x \circ \alpha_{1} y
$$

holds. According to (8), this is equivalent to

$$
\alpha(x-e+y)-\alpha e+e=\alpha x-\alpha e+e-e+\alpha y-\alpha e+e,
$$

that is $\alpha(x-e+y)=\alpha x-\alpha e+\alpha y$. Replacing $x$ with $x+e$ we have

$$
\alpha(x+y)=\alpha(x+e)-\alpha e+\alpha y .
$$

Putting $y=0$, we obtain a relationship $\alpha x=\alpha(x+e)-\alpha e$. This is always true when $\alpha$ is an automorphism of $(Q ;+, 0)$. Therefore, (9) is equivalent to $\alpha(x+y)=\alpha x+\alpha y$.

Independence of left and middle linearity, left, right and middle alinearity concepts with respect to canonical decompositions can be proved in the same way.

\subsection{Canonical decompositions of parastrophes of group iso- topes}

To describe some relation among classes of linear and alinear quasigroups, we have to find a canonical decomposition of every parastrophe of a group isotope. Namely, the following lemma is true. 
Lemma 1. Let $(Q ; \cdot)$ be a group isotope and $(+, 0, \alpha, \beta, a)$ be its canonical decomposition. Then 0-canonical decompositions of all parastrophes of $(Q ; \cdot)$ are the following

$$
\begin{aligned}
& x^{\iota} \cdot y=\alpha x+a+\beta y \\
& \stackrel{s}{x} y=\beta x \stackrel{s}{ } \stackrel{\stackrel{s}{+} \alpha y}{ } \alpha \\
& x^{\ell} \cdot y=\alpha^{-1} x+{ }_{\alpha} J_{\alpha} \alpha^{-1} a+I_{\alpha}^{-1} J_{\alpha} \gamma_{\alpha}^{-1} y \text {; } \\
& x \stackrel{s \ell}{s} y=I_{\alpha} J_{\alpha} \gamma_{\alpha}^{-1} x \underset{\alpha}{\stackrel{s}{+}} J_{\alpha} \alpha^{-1} a \stackrel{s}{+} \alpha^{-1} y \text {; } \\
& x^{r} \cdot y=I_{\beta} J_{\beta} \gamma_{\beta} x+J_{\beta} \beta^{-1} a+\beta_{\beta}^{-1} y ; \\
& x \stackrel{s r}{s} y=\beta^{-1} x+\stackrel{s}{+} J_{\beta} \beta^{-1} a \stackrel{s}{+} I_{\beta}^{-1} J_{\beta} \gamma_{\beta} y,
\end{aligned}
$$

where $\gamma:=\alpha \beta^{-1}, \gamma_{\alpha}:=\alpha^{-1} \gamma \alpha=\gamma_{\beta}:=\beta^{-1} \gamma \beta=\beta^{-1} \alpha$, and according to (7)

$$
I_{\alpha}(x):=J_{\alpha}\left(\alpha^{-1} a\right) \underset{\alpha}{+x}+\alpha^{-1} a, \quad I_{\beta}(x):=J_{\beta}\left(\beta^{-1} a\right) \underset{\beta}{+x}+\beta_{\beta}^{-1} a .
$$

Proof. Since $\alpha 0=\beta 0=0, \stackrel{s}{+}, \underset{\alpha}{+}, \underset{\beta}{+}, \stackrel{s}{+}, \stackrel{s}{+}$ are group operations having 0 as a neutral element. By definition of a canonical decomposition (5), (12) is 0 -canonical decomposition of $s$-parastrophe $\left(Q ;{ }^{s}\right)$. Therefore, to obtain the canonical decomposition of $s$-parastrophe we have to mutually replace 2- and 1-coefficients which are in the canonical decomposition of $(Q ; \cdot)$. That is why $(13)$ and $(15)$ are canonical decompositions of $(\stackrel{s \ell}{\cdot})$ and $(\stackrel{s r}{\cdot})$, if $(12)$ and $(14)$ are canonical decompositions of $(\cdot)$ and $\left({ }^{r} \cdot\right)$.

All coefficients in (12) and (14) are unitary. It remains to prove that (12) and (14) are true. Indeed,

$$
\alpha^{-1} x+\underset{\alpha}{ } J_{\alpha} \alpha^{-1} a+I_{\alpha}^{-1} J_{\alpha} \gamma_{\alpha}^{-1} y \stackrel{(7)}{=} \alpha^{-1}\left(\alpha \alpha^{-1} x+\alpha J_{\alpha} \alpha^{-1} a+I_{\alpha}^{-1} J_{\alpha} \gamma_{\alpha}^{-1} y\right) .
$$

Taking into account the equalities $\alpha J_{\alpha} \alpha^{-1} a=\alpha \alpha^{-1} J \alpha \alpha^{-1} a=J a=-a$ and

$$
\begin{aligned}
& I_{\alpha}^{-1} J_{\alpha} \gamma_{\alpha}^{-1} y=I_{\alpha}^{-1} \alpha^{-1} J \alpha \alpha^{-1} \gamma^{-1} \alpha y=I_{\alpha}^{-1} \alpha^{-1} J \gamma^{-1} \alpha y \\
& \quad=I_{\alpha}^{-1} \alpha^{-1} J \beta \alpha^{-1} \alpha y=I_{\alpha}^{-1} \alpha^{-1} J \beta y=\alpha^{-1} a+\alpha^{-1} J \beta y+{ }_{\alpha} J_{\alpha}\left(\alpha^{-1} a\right) \\
& \quad=\alpha^{-1}\left(a+J \beta y+\alpha J_{\alpha} \alpha^{-1}(a)\right)=\alpha^{-1}(a-\beta y-a),
\end{aligned}
$$


we obtain

$$
\begin{gathered}
\alpha_{\alpha}^{-1} x+J_{\alpha} \alpha^{-1} a+I_{\alpha}^{-1} J_{\alpha} \gamma_{\alpha}^{-1} y=\alpha^{-1}(x-a+a-\beta y-a)= \\
=\alpha^{-1}(x-\beta y-a) \stackrel{(6)}{=} x \stackrel{\ell}{ } \cdot y .
\end{gathered}
$$

Now consider the equality (14):

$$
I_{\beta} J_{\beta} \gamma_{\beta} x+J_{\beta} \beta^{-1} a_{\beta} \beta^{-1} y=\beta^{-1}\left(\beta I_{\beta} J_{\beta} \gamma_{\beta} x+\beta J_{\beta} \beta^{-1} a+\beta \beta^{-1} y\right) .
$$

Since $\beta J_{\beta} \beta^{-1} a=\beta \beta^{-1} J \beta \beta^{-1} a=J a=-a$ and

$$
\begin{aligned}
& \beta I_{\beta} J_{\beta} \gamma_{\beta} x=\beta I_{\beta} \beta^{-1} J \beta \beta^{-1} \alpha x=\beta I_{\beta} \beta^{-1} J \alpha x= \\
& =\beta\left(J_{\beta} \beta^{-1} a+\beta_{\beta}^{-1} J \alpha x+\beta^{-1} a\right)= \\
& =\beta \beta^{-1}\left(\beta J_{\beta} \beta^{-1} a+\beta \beta^{-1} J \alpha x+\beta \beta^{-1} a\right)= \\
& =\beta \beta^{-1} J \beta \beta^{-1} a+J \alpha x+a=J a+J \alpha x+a=-a-\alpha x+a,
\end{aligned}
$$

we have

$$
\begin{gathered}
\beta^{-1}\left(\beta I_{\beta} J_{\beta} \gamma_{\beta} x+\beta J_{\beta} \beta^{-1} a+\beta \beta^{-1} y\right)= \\
=\beta^{-1}(-a-\alpha x+a-a+y)= \\
=\beta^{-1}(-a-\alpha x+y)=x \cdot r \cdot y .
\end{gathered}
$$

The equality (14) can be proved in the same way. Thus, the lemma has been proved.

\subsection{Dependence among linearities}

Theorem 3. Let $i \in\{1,2,3\}$ and $\sigma \in S_{3}$. If a group isotope is $i$-linear ( $i$-alinear), then its $\sigma$-parastrophe is $i \sigma^{-1}$-linear (i $\sigma^{-1}$-alinear).

Proof. Let $(Q ; \cdot)$ be an isotope of a group and let $(10)$ be its canonical decomposition. Lemma 1 implies the following table of components of canonical decompositions of parastrophes of $(Q ; \cdot)$ :

Left and right coefficients are taken from (10)-(15). The middle coefficients are calculated according to their definition. For example, the middle coefficient of $s \ell$-parastrophe is

$$
J_{\alpha} \cdot I_{\alpha} J_{\alpha} \gamma_{\alpha}^{-1} \cdot\left(\alpha^{-1}\right)^{-1}=J_{\alpha}^{2} I_{\alpha} \gamma_{\alpha}^{-1} \alpha=I_{\alpha} \beta_{\alpha}
$$

Using the obtained table, one can calculate linearity of a $\sigma$-parastrophe, if the linearity of $\tau$-parastrophe is known for every $\sigma$ and $\tau$ from $S_{3}$. 


\begin{tabular}{|c|c|c|c|c|c|c|}
\hline \multirow[b]{2}{*}{ Coeffic. } & \multicolumn{5}{|c|}{ Parastrophes } & \multirow[b]{2}{*}{$s r$} \\
\hline & $\iota$ & $s$ & $\ell$ & $s \ell$ & $r$ & \\
\hline Left & $\alpha$ & $\beta$ & $\alpha^{-1}$ & $I_{\alpha} J_{\alpha} \gamma_{\alpha}^{-1}$ & $I_{\beta} J_{\beta} \gamma_{\beta}$ & $\beta^{-1}$ \\
\hline Right & $\beta$ & $\alpha$ & $I_{\alpha}^{-1} J_{\alpha} \gamma_{\alpha}^{-1}$ & $\alpha^{-1}$ & $\beta^{-1}$ & $I_{\beta}^{-1} J_{\beta} \gamma_{\beta}$ \\
\hline Middle & $J \gamma$ & $J \gamma^{-1}$ & $J_{\alpha} \beta_{\alpha}^{-1} J_{\alpha} I_{\alpha}$ & $I_{\alpha} \beta_{\alpha}$ & $I_{\beta} \alpha_{\beta}$ & $J_{\beta} \alpha_{\beta}^{-1} J_{\beta} I_{\beta}$ \\
\hline $\begin{array}{l}\text { Decomp. } \\
\text { group }\end{array}$ & $(+)$ & $(\stackrel{s}{+})$ & $(+)$ & $\left(\begin{array}{c}s \\
+ \\
\alpha\end{array}\right)$ & $(+\underset{\beta}{+})$ & $\left(\begin{array}{c}s \\
+ \\
\beta\end{array}\right)$ \\
\hline
\end{tabular}

TABLE 1. Table of coefficients

For example, let $s \ell$-parastrophe of a group isotope $(Q ; \cdot)$ be middle linear. What linearity has its $r$-parastrophe?

A middle linearity of $s \ell$-parastrophe of $(Q ; \cdot)$ means that its middle coefficient $I_{\alpha} \beta_{\alpha}$ is an automorphism of the group $(Q ; \stackrel{s}{+})$. Since $I_{\alpha}$ is always automorphism of the group, $\beta_{\alpha}$ is an automorphism of $(Q ; \stackrel{s}{+})$. This means that $\beta$ is an automorphism of the group $(Q ;+)$. From the table we see that $r$-parastrophe is right linear.

Full dependence among linearities of parastrophes of group isotopes can be given in the following tables:

\begin{tabular}{|l|l|l|l|l|l|}
\hline$\iota$ & $s$ & $\ell$ & $s \ell$ & $r$ & $s r$ \\
\hline 2 & 1 & 2 & 1 & 3 & 3 \\
\hline 1 & 2 & 3 & 3 & 1 & 2 \\
\hline 3 & 3 & 1 & 2 & 2 & 1 \\
\hline
\end{tabular}

\begin{tabular}{|c|c|c|c|c|c|}
\hline$\iota$ & $s$ & $\ell$ & $s \ell$ & $r$ & $s r$ \\
\hline $2_{a}$ & $1_{a}$ & $2_{a}$ & $1_{a}$ & $3_{a}$ & $3_{a}$ \\
\hline $1_{a}$ & $2_{a}$ & $3_{a}$ & $3_{a}$ & $1_{a}$ & $2_{a}$ \\
\hline $3_{a}$ & $3_{a}$ & $1_{a}$ & $2_{a}$ & $2_{a}$ & $1_{a}$ \\
\hline
\end{tabular}

where $i$ denotes $i$-linearity and $i_{a}$ denotes $i$-alinearity, for all $i=1,2,3$.

On the other hand, each of these numbers can be found using the condition of the theorem. Indeed, let the group isotope be $1_{a}$-linear, i.e., it is 1 -alinear. Then $1(s \ell)^{-1}=1 s r=2 r=3$, therefore $s \ell$-parastrophe is 3 -alinear. The number $3_{a}$ is in the second table in the second row and in the $s \ell$ column. It is easy to verify that this coexistence is in all cases, that is the theorem is true.

The theorem can be reformulated as follows.

Corollary 1. Relationships among single linearity and single alinearity of a group isotope and its parastrophes are the same and can be given in the following table:

\begin{tabular}{|c|c|c|c|c|c|c|}
\hline Linearity & $\iota$ & $\ell$ & $r$ & $s$ & $s \ell$ & $s r$ \\
\hline left & left & left & middle & right & right & middle \\
\hline right & right & middle & right & left & middle & left \\
\hline middle & middle & right & left & middle & left & right \\
\hline
\end{tabular}


The evident result for two-sided linearity of a group isotope and its parastrophes follows from Corollary 1.

Corollary 2. Relationships among two-sided linearity of a group isotope and its parastrophes are the same and can be given in the following table:

\begin{tabular}{|c|c|c|c|c|c|c|}
\hline Linearity & $\iota$ & $\ell$ & $r$ & $s$ & $s \ell$ & $s r$ \\
\hline left-right $(\mathrm{lr})$ & $\mathrm{lr}$ & $\mathrm{lm}$ & $\mathrm{rm}$ & $\mathrm{lr}$ & $\mathrm{rm}$ & $\mathrm{lm}$ \\
\hline left-middle $(\mathrm{lm})$ & $\mathrm{lm}$ & $\mathrm{lr}$ & $\mathrm{lm}$ & $\mathrm{rm}$ & $\mathrm{lr}$ & $\mathrm{rm}$ \\
\hline right-middle $(\mathrm{rm})$ & $\mathrm{rm}$ & $\mathrm{rm}$ & $\mathrm{lr}$ & $\mathrm{lm}$ & $\mathrm{lm}$ & $\mathrm{lr}$ \\
\hline
\end{tabular}

Definition 4. A quasigroup will be called strictly $i$-linear, if it is $i$-linear and it is not $j$-linear for $j \neq i$. A class of quasigroups will be called strictly $i$-linear, if it has a strictly $i$-linear quasigroup.

\section{Linearity bunch}

A parastrophically closed semi-lattice of varieties is called a bunch of varieties. A linearity bunch is a set of varieties which contains the variety of all left linear quasigroups, the variety of all left alinear quasigroups, all their parastrophes and all their possible intersections. Here we prove that the linearity bunch has exactly 14 varieties. 11 of the varieties are well-known and all results (definitions and identities) are summarized in [28].

Let $(Q ; \cdot)$ be a group isotope and $(+, 0, \alpha, \beta, a)$ be its canonical decomposition. Therefore, $\alpha, \beta, J \gamma$ are its left (2-coefficient), right (1-coefficient) and middle (3-coefficient) coefficients, where $\gamma \beta=\alpha$. These relations immediately imply the statements:

- If two coefficients are automorphisms, then the third one is antiautomorphism of the decomposition group;

- If two coefficients are anti-automorphisms, then the third one is anti-automorphism too;

- If a group isotope is $i$-linear and $i_{a}$-linear simultaneously, then the group is commutative, that is the group isotope is central $i$-linear.

Taking into account Theorem 3, Remark 1 and Table 1, we obtain the following relations among all possible types of linearities, where $i$ denotes $i$-linearity, $i_{a}$ denotes $i$-alinearity and $i_{c}$ denotes $i$-centrality, for all $i=1,2,3$ : 


\begin{tabular}{|l|c|c|c|c|c|c|}
\hline Po $(k) /$ Parast. & $\iota$ & $s$ & $\ell$ & $s \ell$ & $r$ & $s r$ \\
\hline semi-linearity & 2 & 1 & 2 & 1 & 3 & 3 \\
\hline semi-alinearity & $2_{a}$ & $1_{a}$ & $2_{a}$ & $1_{a}$ & $3_{a}$ & $3_{a}$ \\
\hline linearity & $23_{a}$ & $23_{a}$ & $21_{a} 3$ & $2_{a} 13$ & $2_{a} 13$ & $21_{a} 3$ \\
\hline alinearity & $2_{a} 1_{a} 3_{a}$ & $2_{a} 1_{a} 3_{a}$ & $2_{a} 1_{a} 3_{a}$ & $2_{a} 1_{a} 3_{a}$ & $2_{a} 1_{a} 3_{a}$ & $2_{a} 1_{a} 3_{a}$ \\
\hline semi-centrality & $2_{c}$ & $1_{c}$ & $2_{c}$ & $1_{c}$ & $3_{c}$ & $3_{c}$ \\
\hline alinearity & $2_{c} 1_{c} 3_{c}$ & $2_{c} 1_{c} 3_{c}$ & $2_{c} 1_{c} 3_{c}$ & $2_{c} 1_{c} 3_{c}$ & $2_{c} 1_{c} 3_{c}$ & $2_{c} 1_{c} 3_{c}$ \\
\hline
\end{tabular}

The Table implies existence of six parastrophic orbits of the corresponding varieties:

- the parastrophic orbit of all semi-linear quasigroups: the variety $\mathfrak{L}_{l}$ of all left linear (i.e., 2-linear) quasigroups, the variety $\mathfrak{L}_{r}$ of all right linear (i.e., 1-linear) quasigroups and the variety $\mathfrak{L}_{m}$ of all middle linear (i.e., 3-linear) quasigroups;

- the parastrophic orbit of all semi-alinear quasigroups: the variety $\mathfrak{L}_{l a}$ of all left alinear (i.e., 2-alinear) quasigroups, the variety $\mathfrak{L}_{r a}$ of all right alinear (i.e., 1-alinear) quasigroups and the variety $\mathfrak{L}_{m a}$ of all middle linear (i.e., 3-alinear) quasigroups;

- the parastrophic orbit of all linear quasigroups: the variety $\mathfrak{L}_{l r}$ of all left-right linear (i.e., 21-linear) quasigroups (traditionally they are called linear quasigroups), the variety $\mathfrak{L}_{l m}$ of all left-middle linear (i.e., 23-linear) quasigroups and the variety $\mathfrak{L}_{r m}$ of all right-middle linear (i.e., 13-linear) quasigroups;

- the parastrophic orbit of all alinear quasigroups has only one variety $\mathfrak{L}_{a}$ which consists of all alinear quasigroups, i.e., each of them is left alinear, right alinear and middle alinear;

- the parastrophic orbit of all semi-central quasigroups: the variety $\mathfrak{L}_{l c}$ of all left central, i.e., $2_{c}$-linear quasigroups (sometimes they are called linear LT-quasigroups), the variety $\mathfrak{L}_{r c}$ of all right central, i.e., $1_{c^{-}}$ linear quasigroups (sometimes they are called linear RT-quasigroups) and the variety $\mathfrak{L}_{m c}$ of all middle central (i.e., $3_{c^{-}}$-alinear) quasigroups;

- the parastrophic orbit of all central quasigroups has only one variety $\mathfrak{L}_{c}$ which consist of liner isotopes of commutative quasigroups, i.e., $T$-quasigroups.

Now, we'll show that all these varieties are different. For this purpose, we will say that a quasigroup $(Q ; *)$ distinguishes a variety $\mathfrak{A}$ if $(Q ; *)$ belongs to $\mathfrak{A}$ and does not belong to these varieties from the linearity bunch which are not included into $\mathfrak{A}$.

Corollary 3. Let $(Q ;+, 0)$ be an arbitrary group, $\alpha, \beta$ be its unitary transformations, i.e., $\alpha 0=\beta 0=0$ and let $(Q ; \circ)$ be defined by $x \circ y:=\alpha x+\beta y$. Then 
1) $(Q ; \circ)$ distinguishes $\mathfrak{L}_{l},(Q ; \stackrel{s}{\circ})$ distinguishes $\mathfrak{L}_{r}$ and $(Q ; \stackrel{r}{\circ})$ distinguishes $\mathfrak{L}_{m}$, if $(Q ;+, 0)$ is not commutative, $\alpha$ is an automorphism of $(Q ;+)$ and $\beta$ is neither automorphism nor anti-automorphism of $(Q ;+, 0)$;

2) $(Q ; \circ)$ distinguishes $\mathfrak{L}_{l a},(Q ; \stackrel{s}{\circ})$ distinguishes $\mathfrak{L}_{r a}$ and $(Q ; \stackrel{r}{\circ})$ distinguishes $\mathfrak{L}_{m a}$, if $(Q ;+, 0)$ is not commutative, $\alpha$ is an anti-automorphism of $(Q ;+)$ and $\beta$ is neither automorphism nor anti-automorphism of $(Q ;+, 0)$;

3) $(Q ; \circ)$ distinguishes $\mathfrak{L}_{l r},(Q ; \stackrel{\ell}{\circ})$ distinguishes $\mathfrak{L}_{l m}$ and $(Q ; \stackrel{r}{\circ})$ distinguishes $\mathfrak{L}_{r m}$, if $(Q ;+, 0)$ is not commutative, $\alpha$ and $\beta$ are automorphisms of $(Q ;+)$;

4) $(Q ; \circ)$ distinguishes $\mathfrak{L}_{a}$, if $(Q ;+, 0)$ is not commutative, $\alpha$ and $\beta$ are anti-automorphisms of $(Q ;+)$;

5) $(Q ; \circ)$ distinguishes $\mathfrak{L}_{l c},(Q ; \stackrel{s}{\circ})$ distinguishes $\mathfrak{L}_{r c}$ and $(Q ; \stackrel{r}{\circ})$ distinguishes $\mathfrak{L}_{m c}$, if $(Q ;+, 0)$ is commutative, $\alpha$ is an automorphism and $\beta$ is not an automorphism of $(Q ;+, 0)$.

Proof. The statement immediately follows from Table 1, where $I_{\alpha}=I_{\beta}=\iota$ because $a=0$. Indeed, let for example $(Q ;+, 0)$ is not commutative, $\alpha$ is an automorphism of $(Q ;+)$ and $\beta$ is neither automorphism nor antiautomorphism of $(Q ;+, 0)$, then left, right and middle coefficients of $s$-parastrophe $(Q ; \stackrel{s}{\circ})$ are $\beta, \alpha$ and $J \beta \alpha^{-1}$ respectively. Therefore, $(Q ; \stackrel{s}{\circ})$ is right linear and neither left nor middle linear. Since the group $(Q ; \stackrel{s}{+}, 0)$ is not commutative, the quasigroup $(Q ; \stackrel{s}{\circ})$ belongs to none of varieties from the linearity bunch except the variety $\mathfrak{L}_{r}$.

From the table follows that left, right and middle coefficients of $r$ parastrophe are equal to $J_{\beta} \gamma_{\beta}, \beta^{-1}$ and $\alpha_{\beta}$ respectively, the decomposition group is $(Q ;+\underset{\beta}{+})$. Taking into account Remark 1 , we conclude that $r$ parastrophe is middle linear and neither left linear, nor right linear, left alinear, right alinear. The group $\left(Q ;{ }_{\beta}^{+}\right)$is not commutative because $(Q ;+)$ is not commutative. Thus, the quasigroup $(Q ; \stackrel{r}{\circ})$ belongs only to variety $\mathfrak{L}_{m}$ from the linearity bunch.

The other items can be proved analogously.

\subsection{The semi-lattice of varieties}

The linearity bunch has 2 totally symmetric and 4 singly symmetric parastrophic orbits. 


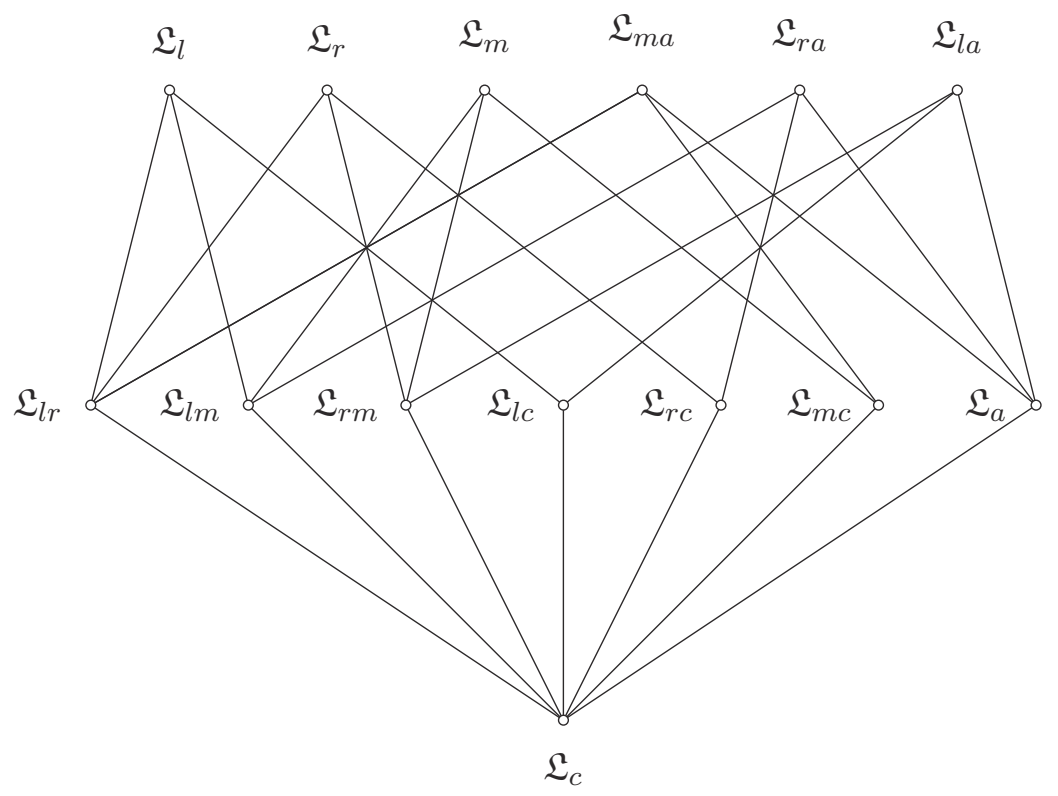

A totally symmetric parastrophic orbit has only one variety. Besides, the variety is totally symmetric, i.e., it coincides with all of its parastrophes, so, its parastrophic symmetry group is $S_{3}$. There are two such varieties in the linearity bunch: the variety of all alinear quasigroups $\mathfrak{L}_{a}$ and the variety of all central quasigroups $\mathfrak{L}_{c}$.

A singly symmetric parastrophic orbit consists of three different pairwise parastrophic varieties. Therefore, each of them has two-element parastrophic symmetry group being a subgroup of $S_{3}$. Since the varieties are parastrophic, their subgroups are conjugate. Thus, the parastrophic symmetry groups are $\{\iota, \ell\},\{\iota, s\},\{\iota, r\}$.

Consider, for example, the set $\left\{\mathfrak{L}_{l}, \mathfrak{L}_{r}, \mathfrak{L}_{m}\right\}$. Since $2 s=1$ and $2 r=3$, Theorem 3 implies ${ }^{s} \mathfrak{L}_{l}=\mathfrak{L}_{r}$ and ${ }^{r} \mathfrak{L}_{l}=\mathfrak{L}_{m}$. Hence, $\left\{\mathfrak{L}_{l}, \mathfrak{L}_{r}, \mathfrak{L}_{m}\right\}$ is a parastrophic orbit. Inasmuch as $2 \ell=2$, the parastrophic symmetry group of $\mathfrak{L}_{l}$ is $\{\iota, \ell\}$ and consequently

$$
\begin{gathered}
\operatorname{Ps}\left(\mathfrak{L}_{r}\right)=r \operatorname{Ps}\left(\mathfrak{L}_{l}\right) r=r\{\iota, \ell\} r=\{\iota, s\}, \\
\operatorname{Ps}\left(\mathfrak{L}_{m}\right)=s \operatorname{Ps}\left(\mathfrak{L}_{l}\right) s=s\{\iota, \ell\} s=\{\iota, r\} .
\end{gathered}
$$

\section{Identities which define varieties in the linearity bunch}

G.B. Belyavskaya and A.Kh. Tabarov have found identities for 11 varieties of the linearity bunch. To obtain all identities it is enough to 
take a collection of identities such that each of them characterizes one variety from every parastrophic orbit. After that consider all parastrophes of the identities. We will find the identities using this method.

\section{The parastrophic orbit of semi-linear quasigroups}

Proposition 2. All semi-linear quasigroups belong to three varieties $\mathfrak{L}_{l}$, $\mathfrak{L}_{r}$ and $\mathfrak{L}_{m}$. These varieties form a singly symmetric parastrophic orbit and they are described by the following pairwise parastrophic identities:

$$
\begin{array}{cc}
\mathfrak{L}_{l}: \quad & x y \cdot z=x\left(u^{r} \cdot u\right) \cdot\left(u^{r} \cdot(u y \cdot z)\right) ; \\
\mathfrak{L}_{r}: \quad\left(z \cdot\left(\left(y^{\ell} \cdot u\right) x\right)=\left(z y^{\ell} \cdot u\right) \cdot\left(\left(u^{\ell} \cdot u\right) x\right) ;\right. \\
\mathfrak{L}_{m}: \quad\left(x^{r} \cdot u y\right) \cdot\left(\left(x^{r} \cdot u^{2}\right)^{r} \cdot u z\right)=y z .
\end{array}
$$

Proof. G.B. Belyavskaya and A.Kh. Tabarov [7] proved that the identity

$$
x\left(u^{r} \cdot y\right) \cdot z=x\left(u^{r} \cdot u\right) \cdot\left(u^{r} \cdot(y \cdot z)\right)
$$

describes the class $\mathfrak{L}_{l}$ of all left linear quasigroups. Replacing $u^{r} \cdot y$ with $y$ and $y$ with $u y$ and applying (1) we obtain (17) which also defines the variety $\mathfrak{L}_{l}$.

By Theorem 1, s-parastrophe of the identity (17) describes the variety $s \mathfrak{L}_{l}$. Since $2 s=1$ and $2(s \ell)^{-1}=2 s r=2 r=1$, Theorem 3 implies that every $s$-parastrophe and every $s \ell$-parastrophe of a left linear quasigroup is right linear, therefore ${ }^{s} \mathfrak{L}_{l}=\mathfrak{L}_{r}={ }^{s \ell} \mathfrak{L}_{l}$.

According to the definition in order to obtain $s$-parastrophe of the identity $(17)$, we have to replace the main operation $(\cdot)$ with $\left({ }^{s}\right)$. As a result, we obtain

$$
\left(x^{s} \cdot y\right) \cdot s=\left[x^{s} \cdot\left(u^{r}\left({ }^{s}\right) u\right)\right] \cdot\left[u^{s}{ }^{r}\left({ }^{s}\right)\left(\left(u^{s} \cdot y\right) \stackrel{s}{s} z\right)\right] .
$$

Using the definition of $s$-parastrophe and applying identities (2) and formula (4), we obtain (18).

By Theorem 1, $r$-parastrophe of the identity (17) describes the variety ${ }^{r} \mathfrak{L}_{l}$. Since $2 r=3$ and $2(s r)^{-1}=2 s \ell=1 \ell=3$, Theorem 3 implies that every $r$-parastrophe and every $s r$-parastrophe of a left linear quasigroup is middle linear, therefore ${ }^{r} \mathfrak{L}_{l}=\mathfrak{L}_{r}={ }^{s r} \mathfrak{L}_{l}$.

According to the definition in order to obtain $r$-parastrophe of the identity $(17)$, we have to replace the main operation $(\cdot)$ with $(\stackrel{r}{r})$. As a result, we obtain

$$
\left(x^{r} \cdot y\right)^{r} \cdot z=\left[x^{r} \cdot\left(u^{r}(\stackrel{r}{r}) u\right)\right]^{r} \cdot\left[u^{r}(\stackrel{r}{r})\left(\left(u^{r} \cdot y\right)^{r} \cdot z\right)\right] .
$$


Applying formula (4) and (3) when $\sigma=r$ :

$$
\left(x^{r} \cdot u^{2}\right) \cdot\left(x^{r} \cdot y\right)^{r} \cdot z=u \cdot\left(\left(u^{r} \cdot y\right)^{r} \cdot z\right) .
$$

Replace $u^{r} \cdot y$ with $y, y$ with $u y$ and use one of $(1)$ :

$$
\left.\left(x^{r} \cdot u^{2}\right) \cdot\left(x^{r} \cdot u y\right)^{r} \cdot z\right)=u \cdot\left(y^{r} \cdot z\right)
$$

Replace $y^{r} \cdot z$ with $z$, use one of (1) and (3) when $\sigma=r$, we obtain (19). The identity defines the variety ${ }^{r} \mathfrak{L}_{l}$, i.e., $\mathfrak{L}_{m}$.

Thus, the parastrophic orbit of semi-linear quasigroups is the set:

$$
\operatorname{Po} \mathfrak{L}_{l}=\left\{\mathfrak{L}_{l}, \mathfrak{L}_{r}, \mathfrak{L}_{m}\right\} .
$$

\section{The parastrophic orbit of semi-alinear quasigroups}

Proposition 3. All semi-alinear quasigroups belong to three varieties $\mathfrak{L}_{l a}$, $\mathfrak{L}_{r a}$ and $\mathfrak{L}_{m a}$. These varieties form a singly symmetric parastrophic orbit and they are described by the following pairwise parastrophic identities:

$$
\begin{array}{cc}
\mathfrak{L}_{l a}: & x\left(y z^{\ell} \cdot u\right)=\left[\left(x\left(u z^{\ell} \cdot u\right)\right)^{\ell} \cdot u\right] y ; \\
\mathfrak{L}_{r a}: & \left(u^{r} \cdot x y\right) z=y\left[u^{r} \cdot\left(\left(u^{r} \cdot x u\right) z\right)\right] ; \\
\mathfrak{L}_{m a}: & \left(u^{\ell} \cdot\left(\left(u^{\ell} \cdot x\right) y\right)\right) u=\left(u^{\ell} \cdot z y\right) \cdot z x .
\end{array}
$$

Proof. G.B. Belyavskaya proved that the identity

$$
\left[u^{r} \cdot\left(\left(x^{\ell} \cdot u\right) y\right)\right] z=y\left[u^{r} \cdot\left(\left(u^{r} \cdot x\right) z\right)\right]
$$

describes the class $\mathfrak{L}_{r a}$ of all right alinear quasigroups (see Theorem 2.2.1 [4]). Replacing $x^{\ell} \cdot u$ with $x$ and $x$ with $x u$ and applying (1), we obtain (21) which also defines the variety $\mathfrak{L}_{r a}$.

By Theorem 1, s-parastrophe of the identity (21) describes the variety ${ }^{s} \mathfrak{L}_{r a}$. Since $1 s=2$ and $1(s r)^{-1}=1 s \ell=2 \ell=2$, Theorem 3 implies that every $s$-parastrophe and every $s r$-parastrophe of a right alinear quasigroup is left alinear, therefore ${ }^{s} \mathfrak{L}_{r a}=\mathfrak{L}_{r a}={ }^{s r} \mathfrak{L}_{r a}$.

According to the definition in order to obtain $s$-parastrophe of the identity $(21)$, we have to replace the main operation $(\cdot)$ with $\left(\cdot{ }^{s}\right)$. Using the definition of $s$-parastrophe and aplying identities (2) and formula (4), we obtain $(20)$.

By Theorem 1, $\ell$-parastrophe of the identity (21) describes the variety ${ }^{\ell} \mathfrak{L}_{r a}$. Since $1 \ell=3$ and $1(s \ell)^{-1}=1 s r=1 \ell s=3 s=3$, Theorem 3 
implies that every $\ell$-parastrophe and every $s r$-parastrophe of a right alinear quasigroup is middle alinear, therefore ${ }^{\ell} \mathfrak{L}_{r a}=\mathfrak{L}_{r a}={ }^{s \ell} \mathfrak{L}_{r a}$.

According to the definition in order to obtain $\ell$-parastrophe of the identity $(21)$, we have to replace the main operation $(\cdot)$ with $(\cdot)$. As a result, we obtain

$$
\left[u^{r}(\stackrel{\ell}{\cdot})\left(x^{\ell} \cdot y\right)\right]^{\ell} \cdot z=y^{\ell} \cdot\left[u^{r}(\stackrel{\ell}{\cdot})\left(\left(u^{r}\left({ }^{\ell}\right)\left(x^{\ell} \cdot u\right)\right)^{\ell} \cdot z\right)\right] .
$$

Applying formula (4), (3) when $\sigma=\ell$ and $r \ell=s r$ :

$$
\left[u^{s r} \cdot\left(x^{\ell} \cdot y\right)\right]^{\ell} \cdot z=y^{\ell} \cdot\left[u^{s r} \cdot\left(\left(u^{s r} \cdot\left(x^{\ell} \cdot u\right)\right)^{\ell} \cdot z\right)\right] .
$$

Using the definition of $s$-parastrophe and aplying identities (2), we obtain:

$$
\left[\left(x^{\ell} \cdot y\right)^{r} \cdot u\right]^{\ell} \cdot z=y^{\ell} \cdot\left[\left(\left(\left(x^{\ell} \cdot u\right)^{r} \cdot u\right)^{\ell} \cdot z\right)^{r} \cdot u\right]
$$

Replace $x^{\ell} \cdot y$ with $x, x$ with $x y$, use one of (1). As the result and by the definition of $\ell$-parastrophe, we obtain:

$$
\left(\left(x^{r} \cdot u\right)^{\ell} \cdot z\right)\left[\left(\left(\left(x y^{\ell} \cdot u\right)^{r} \cdot u\right)^{\ell} \cdot z\right)^{r} \cdot u\right]=y .
$$

Replace $x^{r} \cdot u$ with $u, u$ with $x u$, use one of (1):

$$
\left(u^{\ell} \cdot z\right)\left[\left(\left(\left(x y^{\ell} \cdot x u\right)^{r} \cdot x u\right)^{\ell} \cdot z\right)^{r} \cdot x u\right]=y
$$

Replace $u^{\ell} z$ with $u$, $u$ with $u z$, use one of (1). As the result by the definition of $r$-parastrophe, we have:

$$
u^{r} \cdot y=\left(\left((x y \cdot(x \cdot u z))^{r} \cdot(x \cdot u z)\right)^{\ell} \cdot z\right)^{r} \cdot(x \cdot u z) .
$$

Replace $u^{r} \cdot y$ with $y, y$ with $u y$, use one of (1) and by the definition of $r$-parastrophe:

$$
\left[\left(\left((x \cdot u y)^{\ell} \cdot(x \cdot u z)\right)^{r} \cdot(x \cdot u z)\right)^{\ell} \cdot z\right] y=x \cdot u z .
$$

Replace $x \cdot u z$ with $x, x$ with $x^{\ell} \cdot u z$, use one of (1) and by the definition of $\ell$-parastrophe:

$$
\left(\left(\left(\left(x^{\ell} u z\right) \cdot u y\right)^{\ell} \cdot x\right)^{r} \cdot x\right)^{\ell} \cdot z=x^{\ell} \cdot y
$$


Apply the definition of $\ell$-parastrophe and after $r$-parastrophe:

$$
\left[\left(\left(x^{\ell} \cdot u z\right) \cdot u y\right)^{\ell} \cdot x\right]\left(\left(x^{\ell} \cdot y\right) z\right)=x
$$

Applying the definition of $\ell$-parastrophe twice in a row we obtain (22). The identity defines the variety ${ }^{\ell} \mathfrak{L}_{r a}$, i.e., $\mathfrak{L}_{m a}$.

Thus, the parastrophic orbit of semi-alinear quasigroups is the set:

$$
\operatorname{Po} \mathfrak{L}_{l a}=\left\{\mathfrak{L}_{l a}, \quad \mathfrak{L}_{r a}, \quad \mathfrak{L}_{m a}\right\}
$$

\section{The parastrophic orbit of linear quasigroups}

Proposition 4. All linear quasigroups belong to three varieties $\mathfrak{L}_{l r}, \mathfrak{L}_{l m}$ and $\mathfrak{L}_{r m}$. These varieties form a singly symmetric parastrophic orbit and they are described by the following pairwise parastrophic identities:

$$
\begin{gathered}
\mathfrak{L}_{l r}: x y \cdot u v=x u \cdot\left(\delta_{u} y \cdot v\right), \delta_{u} y:=\left(u^{r} \cdot\left(\left(u^{\ell} \cdot u\right) y \cdot u\right)\right)^{\ell} \cdot\left(u^{r} \cdot u\right) \\
\mathfrak{L}_{l m}: x\left(\delta_{u} y{ }^{\ell} \cdot v\right) \cdot u=x\left(u^{\ell} \cdot v\right) \cdot y, \delta_{u} y:=\left(\left(\left(u^{2}{ }^{\ell} \cdot y\right)^{\ell} \cdot u\right)^{r} \cdot u\right)\left(u^{r} \cdot u\right) \\
\left.\mathfrak{L}_{r m}: \delta_{u} y \cdot\left(\left(y^{\ell} \cdot x\right)^{r} \cdot u\right) v=u \cdot x v, \delta_{u} y:=u^{2} \stackrel{\ell}{\ell}^{\ell}\left(u\left(\left(u^{\ell} \cdot u\right)^{r} \cdot y\right)^{r} \cdot u\right)\right) .
\end{gathered}
$$

Proof. G.B. Belyavskaya proved that the identity (23) describes the class $\mathfrak{L}_{l r}$ of all linear quasigroups (see Theorem 2.3.1 [4]).

By Theorem 1 and Corollary 2, $\ell$-parastrophe of the identity (23) describes the variety ${ }^{\ell} \mathfrak{L}_{l r}$. Since $1 \ell=3,1(s \ell)^{-1}=1 s r=1 \ell s=3 s=3$, Theorem 3 implies that every $\ell$-parastrophe and every $s \ell$-parastrophe of a left-right linear quasigroup is left-middle linear, therefore ${ }^{\ell} \mathfrak{L}_{l r}=\mathfrak{L}_{l r}=$ ${ }^{s \ell} \mathfrak{L}_{l r}$.

According to the definition in order to obtain $\ell$-parastrophe of the identity $(23)$, we have to replace the main operation $(\cdot)$ with $(\cdot)$ :

$$
\begin{gathered}
\left(x^{\ell} \cdot y\right)^{\ell} \cdot\left(u^{\ell} \cdot v\right)=\left(x^{\ell} \cdot u\right)^{\ell} \cdot\left(\delta_{u} y^{\ell} \cdot v\right), \\
\left.\delta_{u} y=\left[u^{r}(\stackrel{\ell}{\bullet})\left(\left(u^{\ell}(\stackrel{\ell}{\cdot}) u\right)^{\ell} \cdot y\right)^{\ell} \cdot u\right)\right]^{\ell}(\stackrel{\ell}{\cdot})\left(u^{r}(\stackrel{\ell}{\bullet}) u\right) .
\end{gathered}
$$

Replace $x^{\ell} \cdot y$ with $x, x$ with $x y$, use one of (1) and apply formula (4), (3) when $\sigma=\ell$ and $r \ell=s r$ :

$$
x^{\ell} \cdot\left(u^{\ell} \cdot v\right)=\left(x y^{\ell} \cdot u\right)^{\ell}\left(\delta_{u} y^{\ell} \cdot v\right), \quad \delta_{u} y=\left[u^{s r} \cdot\left(\left(u^{2} \cdot y\right)^{\ell} \cdot u\right)\right] \cdot\left(u^{s r} \cdot u\right) .
$$


Replace $u^{\ell} \cdot v$ with $u$, $u$ with $u v$, use one of (1) and apply the definition of $s$-parastrophe:

$\left.x^{\ell} \cdot u=\left(x y{ }^{\ell} \cdot u v\right)^{\ell} \cdot\left(\delta_{u v} y^{\ell} \cdot v\right), \quad \delta_{u v} y=\left[\left((u v)^{2} \cdot y\right)^{\ell} \cdot u v\right)^{r} \cdot u v\right] \cdot\left(u v^{r} \cdot u v\right)$.

Replace $x^{\ell} \cdot u$ with $x, x$ with $x u$, use one of (1) and apply the definition of $\ell$-parastrophe:

$$
\left.(x u \cdot y)^{\ell} \cdot u v=x\left(\delta_{u v} y^{\ell} \cdot v\right), \quad \delta_{u v} y=\left[\left((u v)^{2} \cdot y\right)^{\ell} \cdot u v\right)^{r} \cdot u v\right] \cdot\left(u v^{r} \cdot u v\right) .
$$

Replace $u v$ with $u, u$ with $u^{\ell} \cdot v$, use one of (1) and apply the definition of $\ell$-parastrophe. As the result, we obtain (24). The identity defines the variety ${ }^{\ell} \mathfrak{L}_{l r}$, i.e., $\mathfrak{L}_{l m}$.

By Theorem 1 and Corollary 2, r-parastrophe of the identity (23) describes the variety ${ }^{r} \mathfrak{L}_{l r}$. Since $2 r=3,2(s r)^{-1}=2 s \ell=2 r s=3 s=3$, Theorem 3 implies that every $r$-parastrophe and every $s r$-parastrophe of a left-right linear quasigroup is right-middle linear, therefore ${ }^{r} \mathfrak{L}_{l r}=\mathfrak{L}_{l r}=$ ${ }^{s r} \mathfrak{L}_{l r}$.

According to the definition in order to obtain $r$-parastrophe of the identity $(23)$, we have to replace the main operation $(\cdot)$ with $(\stackrel{r}{\cdot})$ :

$$
\begin{gathered}
\left(x^{r} \cdot y\right)^{r} \cdot\left(u^{r} \cdot v\right)=\left(x^{r} \cdot u\right)^{r} \cdot\left(\delta_{u} y^{r} \cdot v\right), \\
\left.\delta_{u} y=\left[u^{r} \stackrel{r}{ }{ }^{r}\right)\left(\left(\left(u^{\ell} \stackrel{r}{ }(\cdot) u\right)^{r} \cdot y\right)^{r} \cdot u\right)\right] \stackrel{\ell}{ }(\stackrel{r}{\cdot})\left(u^{r} \stackrel{r}{ }(\cdot) u\right) .
\end{gathered}
$$

Replace $u^{r} \cdot v$ with $v, v$ with $u v$, use one of (1) and apply formula (4), (3) when $\sigma=r$ and $\ell r=s \ell$ :

$$
\left(x^{r} \cdot y\right)^{r} \cdot v=\left(x^{r} \cdot u\right)^{r} \cdot\left(\delta_{u} y^{r} \cdot u v\right), \quad \delta_{u} y=\left[u \cdot\left(\left(\left(u^{s \ell} \cdot u\right)^{r} \cdot y\right)^{r} \cdot u\right)\right]^{s \ell} \cdot u^{2} .
$$

Replace $x^{r} \cdot u$ with $u, u$ with $x u$, use one of (1) and apply the definition of $s$-parastrophe:

$$
\left(x^{r} \cdot y\right)^{r} \cdot v=u^{r} \cdot\left(\delta_{x u} y^{r} \cdot(x u \cdot v)\right), \delta_{x u} y=(x u)^{2} \cdot\left[x u \cdot\left(\left(\left(x u^{\ell} \cdot x u\right)^{r} \cdot y\right)^{r} \cdot x u\right)\right] .
$$

Use the definition of $r$-parastrophe twice in a row and replace $x^{r} \cdot y$ with $y, y$ with $x y$, use one of (1):

$$
\delta_{x u} x y \cdot u\left(y^{r} \cdot v\right)=x u \cdot v, \quad \delta_{x u} x y=(x u)^{2} \cdot\left[x u \cdot\left(\left(\left(x u^{\ell} \cdot x u\right)^{r} \cdot x y\right)^{r} \cdot x u\right)\right] .
$$


Replace $y^{r} \cdot v$ with $v, v$ with $y v$, use one of (1):

$$
\delta_{x u} x y \cdot u v=x u \cdot y v, \quad \delta_{x u} x y=(x u)^{2}{ }^{\ell} \cdot\left[x u \cdot\left(\left(\left(x u^{\ell} \cdot x u\right)^{r} \cdot x y\right)^{r} \cdot x u\right)\right] .
$$

Replacing $x u$ with $u, u$ with $x^{r} \cdot u$ and replacing $x y$ with $y, y$ with $x^{r} \cdot y$ using one of (1), we obtain (25). The identity defines the variety ${ }^{r} \mathfrak{L}_{l r}$, i.e., $\mathfrak{L}_{r m}$.

Thus, the parastrophic orbit of linear quasigroups is the set:

$$
\operatorname{Po} \mathfrak{L}_{l r}=\left\{\mathfrak{L}_{l r}, \mathfrak{L}_{l m}, \mathfrak{L}_{r m}\right\} .
$$

\section{The parastrophic orbit of semi-central quasigroups}

Proposition 5. All semi-central quasigroups belong to three varieties $\mathfrak{L}_{l c}$, $\mathfrak{L}_{r c}$ and $\mathfrak{L}_{m c}$. These varieties form a singly symmetric parastrophic orbit and they are described by the following pairwise parastrophic systems of identities:

$$
\begin{aligned}
\mathfrak{L}_{l c}: & \left\{\begin{array}{l}
x y \cdot z=x\left(u^{r} \cdot u\right) \cdot\left(u{ }^{r} \cdot(u y \cdot z)\right), \\
x(y \cdot u v)=u(y \cdot x v) ;
\end{array}\right. \\
\mathfrak{L}_{r c}: & \left\{\begin{array}{l}
x \cdot y z=\left((x \cdot y u)^{\ell} \cdot u\right) \cdot\left(u^{\ell} \cdot u\right) z, \\
x\left(y^{r} \cdot u v\right)=u\left(y^{r} \cdot x v\right) ;
\end{array}\right. \\
\mathfrak{L}_{m c}: & \left\{\begin{array}{l}
u\left(\left(u^{r} \cdot x y\right)^{r} \cdot y z\right)=\left(x^{r} \cdot u^{2}\right) z, \\
\left.x\left(y^{r} \cdot u v\right)=u(y)^{r} \cdot x v\right)
\end{array}\right.
\end{aligned}
$$

Proof. A.Kh. Tabarov proved that the systems of identities

$$
\begin{aligned}
& \left\{\begin{array}{l}
x\left(u^{r} \cdot y\right) \cdot z=x\left(u^{r} \cdot u\right) \cdot\left(u^{r} \cdot y z\right), \\
x^{r} \cdot\left(y \cdot\left(u^{r} \cdot v\right)\right)=u^{r} \cdot\left(y \cdot\left(x^{r} \cdot v\right)\right) ;
\end{array}\right. \\
& \left\{\begin{array}{l}
x \cdot\left(y^{\ell} \cdot u\right) z=\left(x y^{\ell} \cdot u\right) \cdot\left(u^{\ell} \cdot u\right) z, \\
x^{r} \cdot\left(y \cdot\left(u^{r} \cdot v\right)\right)=u^{r} \cdot\left(y \cdot\left(x^{r} \cdot v\right)\right) ;
\end{array}\right.
\end{aligned}
$$

described the class $\mathfrak{L}_{l c}$ of all left central quasigroups and the class $\mathfrak{L}_{r c}$ of all right central quasigroups respectively (see Theorem 2.6.1 [28]). V.D. Belousov [1] found the second identity in each of these systems. This identity defines the variety of all quasigroups which are isotopic to commutative groups. We shall present the given identity in a more simpler form by primarily-equivalent transformations, namely replacing $x^{r} \cdot v$ with $v, v$ with $x v$, using one of (1) and applying the definition of $r$-parastrophe, 
obtaining the second identity in every system (26), (27) and (28). In the first identity of the first system we replace $u^{r} \cdot y$ with $y, y$ with $u y$ and in the first identity of the second system we replace $y^{\ell} \cdot u$ with $y, y$ with $y u$ using one of (1). Consequently, we obtain the identities from systems (26) and (27) respectively.

By Theorem 1 and Corollary 2,s-parastrophe of the first identity from(26) describes the variety ${ }^{s} \mathfrak{L}_{l c}$. Since $2 s=1,2(s \ell)^{-1}=2 s r=1 r=1$, Theorem 3 implies that every $s$-parastrophe and every $s \ell$-parastrophe of a left central quasigroup is right central, so ${ }^{s} \mathfrak{L}_{l c}=\mathfrak{L}_{r c}={ }^{s \ell} \mathfrak{L}_{l c}$.

By Theorem 1 and Corollary 2, $r$-parastrophe of the first identity from (26) describes the variety ${ }^{r} \mathfrak{L}_{l c}$. Since $2 r=3$ and $2(s r)^{-1}=2 s \ell=1 \ell=3$, Theorem 3 implies that every $r$-parastrophe and every $s r$-parastrophe of a left central quasigroup is middle central, therefore ${ }^{r} \mathfrak{L}_{l c}=\mathfrak{L}_{m c}={ }^{s r} \mathfrak{L}_{l c}$.

According to the definition in order to obtain $r$-parastrophe of the first identity from $(26)$, we have to replace the main operation $(\cdot)$ with $\left({ }^{r}\right)$ :

$$
\left(x^{r} \cdot y\right)^{r} \cdot z=\left[x^{r} \cdot\left(u^{r}(\stackrel{r}{\cdot}) u\right)\right]^{r} \cdot\left[u^{r}(\stackrel{r}{r})\left(\left(u^{r} \cdot y\right)^{r} \cdot z\right)\right] .
$$

Applying formula (4), (3) when $\sigma=r$, we obtain:

$$
\left(x^{r} \cdot y\right)^{r} \cdot z=\left(x^{r} \cdot u^{2}\right)^{r} \cdot\left[u\left(\left(u^{r} \cdot y\right)^{r} \cdot z\right)\right] .
$$

Replace $x^{r} \cdot y$ with $y, y$ with $x y$, use one of (1) and apply definition of $r$-parastrophe:

$$
\left(x^{r} \cdot u^{2}\right) \cdot\left(y^{r} \cdot z\right)=u \cdot\left(\left(u^{r} \cdot y\right)^{r} \cdot z\right) .
$$

Replace $y^{r} \cdot z$ with $z, z$ with $y z$, use one of (1) and apply definition of $r$-parastrophe:

$$
u^{r} \cdot\left(x^{r} \cdot u^{2}\right) z=\left(u^{r} \cdot y\right)^{r} \cdot y z .
$$

Using the definition of $r$-parastrophe, we obtain the first identity from (28). The identity defines the variety ${ }^{r} \mathfrak{L}_{l c}$, i.e., $\mathfrak{L}_{m c}$.

Thus, the parastrophic orbit of semi-central quasigroups is the set:

$$
\operatorname{Po} \mathfrak{L}_{l c}=\left\{\mathfrak{L}_{l c}, \mathfrak{L}_{r c}, \mathfrak{L}_{m c}\right\} .
$$

\section{The parastrophic orbit of alinear quasigroups}

Proposition 6. All alinear quasigroups belong to one variety $\mathfrak{L}_{a}$. This variety forms a totally symmetric parastrophic orbit and it is described by the following identity:

$$
\mathfrak{L}_{a}: \quad x y \cdot u v=\left(\delta_{x} v \cdot y\right) \cdot u x, \quad \delta_{x} v:=\left[\left(x \cdot\left(x^{r} \cdot x\right) v\right)^{\ell} \cdot x\right]^{\ell} \cdot\left(x^{r} \cdot x\right) .
$$


Proof. G.B. Belyavskaya proved that the identity (29) describes the class $\mathfrak{L}_{a}$ of all alinear quasigroups (see Theorem 2.3.2 [4]). The variety is totally symmetric because if two coefficients are anti-automorphisms then the third one is also an anti-automorphism.

\section{The parastrophic orbit of central quasigroups}

Proposition 7. All central quasigroups belong to one variety $\mathfrak{L}_{c}$. This variety forms a totally symmetric parastrophic orbit and it is described in the following system of identities:

$$
\left\{\begin{array}{l}
x y \cdot z=x\left(u^{r} \cdot u\right) \cdot\left(u^{r} \cdot(u y \cdot z)\right), \\
x \cdot y z=\left((x \cdot y u)^{\ell} \cdot u\right) \cdot\left(u^{\ell} \cdot u\right) z, \\
x\left(y^{r} \cdot u v\right)=u\left(y^{r} \cdot x v\right)
\end{array}\right.
$$

Proof. A variety of central quasigroups is a intersection of varieties of linear and alinear quasigroups. The fact is stated by G.B. Belyavskaya (see the Corollary 2.4.2 [4]). The variety is totally symmetric because if a decomposition group is commutative and two coefficients are automorphisms then the third one is also an automorphism.

\section{Conclusion}

The results and methods of group theory are effectively generalized on the quasigroups which are close to groups. Moufang loops are closest to groups in the class of loops, and linear, central and medial quasigroups are closest to groups and commutative groups in the class of quasigroups. On the other hand, isotopy changes almost all algebraic concepts so much that they disappear in the respective isotopes.

This full classification of quasigroups with some linearity properties allows to solve the problems in quasigroup theory, especially to construct the necessary examples.

\section{Acknowledgements}

The authors are grateful to the members of Scientific Ukrainian Mathematical School "Multiary Invertible Functions" (SUMS "MIF") for their helpful discussions on the problem and to the English reviewer V. Obshanska. 


\section{References}

[1] V.D. Belousov Balanced identities in quasigroups, Matem. zbornik, vol. 70 no. 1(112), 1966, pp. 55-97.

[2] V.D. Belousov Foundation of the theory of quasigroups and loops, M.: Nauka, 1967, 222p. (in Russian).

[3] G.B. Belyavskaya Abelian quasigroups are T-quasigroups, Quasigroups and related systems, Vol. 1, no. 1, 1994, pp. 1-7.

[4] G.B.Belyavskaya Quasigroups: Identities with permutations, linearity and nuclei, LAP, Germany, 2013. (in Russian).

[5] G.B.Belyavskaya Parastrophically equivalent identities characterizing quasigroups isotopic to abelian groups, Quasigroups and related systems, Vol. 1, no. 22, 2014, pp. 19-32.

[6] G.B. Belyavskaya, A.Kh. Tabarov Homomorphisms and endomorphisms of linear and alinear quasigroups, Discrete Math. Appl. 17, no.3, 2007, pp. 253-260.

[7] G.B.Belyavskaya, A.Kh.Tabarov Characteristic of linear and alinear quasigroups, Diskretnaya matematika, RAN, Moscow, Tom 4, vyp.2, 1992, pp. 142-147. (in Russian).

[8] G.B.Belyavskaya, T-quasigroups and center of quasigroups, Matem. issledov. Kishinev:Ştiinţa, Vyp. III, 1989, pp. 24-43.

[9] R. Freese, R. McKenzie Commutator Theory for Congruence Modular Varieties, London Math. Soc. Lecture Notes, Ser. 125, 1987.

[10] Jan Galuszka Codes of groupoids with one-sided quasigroup conditions, J. Algebra and discrete mathematics, Vol 8, No 2, 2009, pp. 27-44.

[11] Jan Galuszka Lattices of classes of groupoids with one-sided quasigroup conditions, J. Algebra and discrete mathematics, Vol 9, No 1, 2010, pp. 31-40.

[12] J. Jezek and T. Kepka Quasigroups isotopic to a group, Comment Math. Univ. Carol, no. 1(16), 1975, pp. 59-76.

[13] T. Kepka, P. Němec T-quasigroups. I., Acta Univ. Carol., Math. Phys. 12, no. 1, 1971, pp. 39-49.

[14] T. Kepka, P. Němec T-quasigroups. II., Acta Univ. Carol., Math. Phys. 12, no. 2, 1971, pp. 31-49.

[15] H.V. Krainichuk Classification of group isotopes according to their symmetry groups, Folia Mathematica, Vol. 19, No. 1, Acta Universitatis Lodziensis, Poland, 2017, pp. 84-98.

[16] Alberto Marini, Victor Shcherbacov On autotopies and automorphisms of n-ary linear quasigroups, J. Algebra and discrete mathematics, Vol 3, No 2, 2004, pp. 59-83.

[17] V.A. Shcherbacov Elements of Quasigroup Theory and Applications, Chapman and Hall/CRC, 2017.

[18] J.D.H. Smith Groups, triality and hyperquasigroups, J. Pure Appl. Algebra, 216:4, 2012, pp. 811-825.

[19] F.M. Sokhatsky On clasification of functional equations on quasigroups, Ukrainian Math. Journal. - .- vol. 56, no. 9, 2004, pp. 1259-1266. (in Ukrainian). 
[20] F.M. Sokhatsky Parastrophic symmetry in quasigroup theory, Visnyk DonNU, Ser. A. Natural Sciences: Math., 2016, pp.72-85.

[21] F.M. Sokhatsky Symmetry in quasigroup and loop theory, 3rd Mile High Conf. on Nonassoc. Math., Denver, Colorado, USA, August 11-17, 2013.

[22] F.N. Sokhatskii About group isotopes I., Ukrainian Math. J., 47(10), 1995, pp. $1585-1598$.

[23] F.N. Sokhatskii About of group isotopes II., Ukrainian Math. J., 47(12), 1995, pp. 1935-1948.

[24] F.N. Sokhatskii About of group isotopes III., Ukrainian Math. J., 48(2), 1996, pp. $251-259$.

[25] F. Sokhatskyj, P. Syvakivskyj On linear isotopes of cyclic groups, Quasigroups and related systems, Vol. 1, no. 1, 1994, pp. 66-76.

[26] David Stanovský, Petr Vojtěchovský Central and medial quasigroups of small order, Bul. Acad. Ştiinţe Repub. Mold. Mat., no. 1(80), 2016, pp. 24-40.

[27] Sushkevich A.K. Generalized group theory, Kyiv, 1937.

[28] A.Kh. Tabarov Identities and linearity of quasigroups, Dissertation for the degree of Doctor in Physical and Mathematical Sciences, Moscow, Moscow State University, 2009. (in Russian)

[29] A.Kh. Tabarov Kernels, Linearity and Balanced Identities in Quasigroups, Dissertation for the degree of Candidate in Physical and Mathematical Sciences, Chisinau, Academy of Sciences of the Republic of Moldova, Institute of Mathematics, 1992. (in Russian)

\section{CONTACT INFORMATION}

Fedir M. Sokhatsky Faculty of Information and Applied Technologies, Department of Applied Mathematics, Vasyl' Stus Donetsk National University, Vinnytsia, 21021, Ukraine E-Mail(s): fmsokha@ukr.net

Halyna V. Krainichuk

Volodymyr A. Sydoruk
Faculty of Information and Applied Technologies, Department of radiophysics and cybersecurity, Vasyl' Stus Donetsk National University, Vinnytsia, 21021, Ukraine E-Mail(s): kraynichuk@ukr.net

Tyvriv Boarding School, Tyvriv, 23300, Ukraine E-Mail(s): ssvvaa1@gmail.com

Received by the editors: 28.12 .2020

and in final form 05.06.2021. 